\title{
Exploiting Delay Budget Flexibility for Efficient Group Delivery in the Internet of Things
}

\author{
Yuhui Yao, Yan Sun, Chris Phillips, Yue Cao, Member, IEEE and Jichun Li
}

\begin{abstract}
Further accelerated by the Internet of Things (IoT) concept, various devices are being continuously introduced into diverse application scenarios. To achieve unattended updates of IoT smart object(s), there remains a challenging problem concerning how to efficiently deliver messages to specific groups of target nodes, especially considering node mobility. In this paper, the relay selection problem is investigated on the basis of directional movement with randomness (e.g. typically associated with the searching or migrating behaviour of animals). Unlike numerous works tackling one-to-one communication, we focus on efficient group delivery (one-to-many). A two-level delay budget model is considered to reflect the flexibility of delay tolerance, which brings potential efficiency gains for group delivery compared with using a single budget boundary. Following the description of the system model, a combinatorial bi-objective optimisation problem is formulated and solutions are proposed. Simulation results show that the greedy algorithm can achieve comparable performance to an evolutionary algorithm when the delivery satisfaction outweighs efficiency. Furthermore, we show that our proposed greedy scheme can outperform the state-of-theart when the delivery efficiency becomes increasingly important.
\end{abstract}

Index Terms-Internet of Things, group delivery, relay selection, delay budget, directional movement

\section{INTRODUCTION}

Along with the fast evolution of information technologies, Internet services have now widely penetrated into our daily lives. Further accelerated by the Internet of Things (IoT) concept, various devices are being continuously introduced into diverse application scenarios that promise a ubiquitous means to sense and control everything [1]. In contrast with the conventional Internet, a network node in this IoT context could be different types of device embedded with network interface(s), such as intelligent appliances [2], multimedia sensors [3], and robotic actuators [4]. Within the context of IoT, the term 'smart object' (interchangeably used with the term 'node' in the remainder of this paper) is used to represent any possible thing that can provide data and/or undertake actions for IoT applications [5].

For long-term continuous operation of IoT systems, reconfigurations and upgrades are usually inevitable. As discussed in [6], it has been realised that the unattended updating of smart object(s) can be achieved by disseminating a package of updated instructions (called a code package). Without loss

Y. Yao, Y. Sun, and C. Phillips are with the Department of Electronic Engineering and Computer Science, Queen Mary University of London, London E1 4NS, U.K. (e-mail: yuhui.yao@qmul.ac.uk; yan.sun@qmul.ac.uk; chris.i.phillips@qmul.ac.uk)

Y. Cao is with the School of Computing and Communications, Lancaster University, Lancaster LA1 4WA, U.K. (e-mail: yue.cao@lancaster.ac.uk)

$\mathrm{J.Li}$ is with the Department of Engineering, Teesside University, Middlesbrough TS1 3BX, U.K. (e-mail: j.li@tees.ac.uk) of generality, this paper assumes that code packages are sent from the network gateway that serves as an interface to outside networks. Because IoT smart objects are typically batterypowered and rely on wireless multi-hop communications [7], blind broadcasting/flooding is not a feasible way of package delivery especially when some packages have a certain tolerance of delivery failure ${ }^{1}$ and are only aimed at a subset of the nodes. Given specific package targets, how to efficiently perform group delivery remains a challenging problem (especially considering mobility), which is the research focus of this paper.

It is worth noting that delayed delivery of a code package could be acceptable in many cases. Larger delay budgets can result from either non-urgent packages or planned updates (e.g. scheduled minutes or even hours in advance). Based on research concerning Delay/Disruption Tolerant Networks (DTNs) [8] and Mobile Opportunistic Networks (MONs) [9], it is possible to facilitate the dissemination of delay-tolerant information by making full use of node mobility. However, as suggested in [8], most of the existing studies are limited to individual deliveries where data is destined for a single destination, and thus more studies are needed to address multidestination group delivery.

Based on knowledge of vehicular navigation, delay-tolerant delivery has been utilised to reduce forwarding hops in the literature such as [10] and [11]. Nevertheless, the exact movement trajectory of mobile nodes can hardly be known in advance with regard to some IoT scenarios (e.g. a sensor deployed upon animals). Although our previous work [12] has provided an analysis of directional movement (e.g. foraging or migrating behaviour of animals [13]) for the delivery to individual destinations, it has hitherto been an open issue regarding how to take advantage of such random movements with directional correlation for the group delivery.

In this paper, feasible exploitation of directional movement is further investigated for efficient group delivery based on the contact prediction model proposed in [12]. Compared with the individual delivery, group delivery brings more optimisation opportunities (relay sharing between targets) but introduces more complexity (in terms of metrics and algorithms) for making decisions. Considering delivery efficiency and reliability, suitable selection of relay node(s) is a key challenge for group delivery based on the delay-tolerant routing. With an

\footnotetext{
${ }^{1}$ Failure tolerance is related to factors such as package importance and/or backup schemes. Possible backup schemes for delivery failure are out-of-scope for this paper but could be researched in the future.
} 
awareness of directional correlation ${ }^{2}$, it is possible to leverage random movement for optimised group relay selection, which is beneficial for reducing energy consumption and prolonging system lifetime. The main contributions of this paper are listed as follows:

- An analytical framework is proposed to formulate the group relay selection as a combinatorial bi-objective optimisation problem. Our research considers a two-level delay budget model to reflect the flexibility of delay tolerance, which allows more optimisation opportunities whilst maintaining delivery satisfaction within acceptable bounds.

- Both an evolutionary approach and a greedy approach are proposed to solve the formulated problem. Our proposed evolutionary algorithm reveals a trade-off between delivery satisfaction and cost reduction, which approximates a theoretical limit of delivery performance. Our greedy algorithm can make better decisions than the existing stateof-the-art to improve group delivery efficiency because the two-level delay budget is considered in group relay selection.

The rest of this paper is organized as follows. In Section II, related works are reviewed and the research motivation is summarised. In Section III, the system model is described for later problem formulation and evaluation. In Section IV, the research problem is formulated and the proposed solutions are demonstrated. In Section V, the performance of proposed solutions are further analysed and discussed. The paper finally concludes in Section VI.

\section{BACKGROUND AND MOTIVATION}

\section{A. Delivery Strategy}

As surveyed in [8], the Store-Carry-Forward (SCF) strategy has been widely researched for routing in DTNs. Two simplest SCF schemes are: Direct Delivery (DD) [14], which requires no replication and source node keeps the message until the destination node is in proximity; and Epidemic [15], which blindly replicates the message to every encountered node without relay selection. Despite their simplicity, these two basic schemes are usually not enough to meet the efficiency/reliability of delivery requirements so many later works have been proposed for replication control and relay selection, such as [16], [17], [18].

In literature, a Forward-Wait-Deliver (FWD) strategy has been proposed for efficient delivery [12]. The FWD strategy delivers a package in three steps:

- Forward to stationary relay;

- Wait for mobile target;

- Deliver when target contacts the relay.

Similar to SCF routing, the FWD strategy relies on opportunistic contacts resulting from node mobility. However, the FWD scheme is also supported by the multi-hop forwarding over connectible nodes and the research focus is to achieve

\footnotetext{
${ }^{2}$ The directional correlation means the temporal dependency of movement direction for each individual node. The correlation of movement direction between different nodes is beyond the scope of this paper.
}

delivery by the shortest-possible forwarding route. To this end, FWD research is distinct from the traditional DTN works and is aimed at multi-hop wireless networking scenarios.

Compared with the approach of immediate delivery (via a multi-hop route) to the package targets, shorter routes (i.e. fewer hops) are most likely to be employed by the FWD strategy, as it makes the full use of predictable contacts. Consequently, the FWD strategy brings a potential efficiency gain given tolerance of delivery delay. In [10], the FWD strategy is adopted in a scenario where sensors send data to a mobile sink moving according to a predefined trajectory. The feasibility of the FWD strategy is further investigated in [12] on the basis of directional movement with randomness. However, it is worth noting that the possibility of relay sharing is overlooked in the aforementioned works as each individual delivery is assumed to be independent.

\section{B. Group Delivery}

In some recent works such as [19], [20], [11], the adoption of the FWD strategy has been investigated for group delivery. With the FWD strategy, it attempts to identify a shareable relay for a (sub)group of targets, which further reduces transmission times compared with treating each delivery separately. In [19] and [20], the storage constraint is considered to maximise the overall rate of successful delivery. In [11], the maximum acceptable delay is given to minimise the overall forwarding cost. However, these approaches all rely on exact knowledge of the vehicular route in advance. Consequently, they are not directly applicable to the scenarios where mobile targets do not strictly follow predefined trajectories, such as animals in the wild.

With regard to partially predictable mobility, further studies are required to apply the FWD strategy for efficient group delivery. Specifically, our research focus is on the scenario where mobile nodes (e.g. deployed on animals) tend to move with more-or-less directional correlation (e.g. searching or migrating behaviour) while their movement trajectories cannot be exactly predicted. In [12], the directional correlation of random movement has been investigated for efficient delivery. However, considering optimised group relay selection, the feasible exploitation of directional movement for group delivery is still an unsolved problem.

To exploit the optimisation opportunities in group delivery, algorithms are required to be designed. As proved in [19] and [11], the group relay selection problem is at least NP-hard (i.e. non-deterministic polynomial-time hardness) and it is infeasible to search for an optimal solution [21]. Heuristic algorithms are therefore proposed to find an approximation to the optimum in a reasonably short time [22]. As a typical paradigm of heuristic algorithms, the greedy algorithm is commonly considered to solve the group relay selection problem, such as in [19], [20], [11]. However, none of these existing works have evaluated the performance of their greedy algorithms in multi-objective optimisation.

To this end, we propose to further investigate the feasibility of the FWD strategy for efficient group delivery. Based on directional correlation of random movements, the opportunity 
for relay sharing can be exploited to reduce the overall forwarding hops. We make the first attempt to provide such an analytical framework for group relay selection. Over many literature works on the topological routing, our work also contributes to the group delivery based on the geographic routing (as node positions are considered for the contact prediction), which is an issue overlooked by the existing research [23]. Following the system model described in the next section, our proposal will be further explained.

\section{SYSTEM MODEL}

\section{A. Overview}

In this section, the group delivery system model is described from three aspects. Firstly, the package targets and candidate relays are specified in Section III-B. Then a two-level delay budget model is discussed in Section III-C as the delivery requirement. Finally, the contact prediction model is briefly introduced from [12] in Section III-D. Although this paper mainly takes habitat monitoring [24] as an example IoT system, the application of our research can be generalised to other similar scenarios that involve directional movement with randomness.

An illustrative example of the group delivery system is provided in Figure 1 where three mobile nodes are the delivery targets and twenty-five stationary nodes are the candidate relays. With the consideration of acceptable delivery delay, one or more candidate relays are to be selected as the relay node, to deliver a package from the sink node to target nodes. Based on contact prediction, the package is forwarded to the selected relay for later delivery when the target node is directly contactable. In this way, fewer overall forwarding hops need to be employed leading to more efficient group delivery, as the feasible opportunities of relay sharing can be exploited.

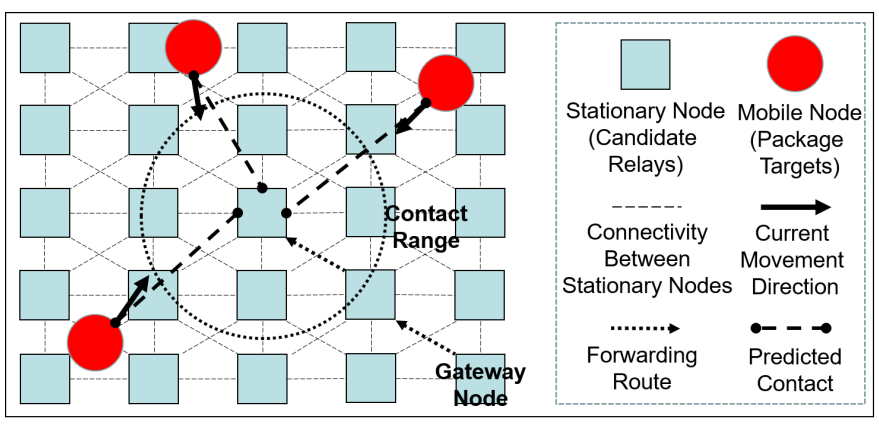

Fig. 1. Example scenario of the group delivery system

\section{B. Node Deployment}

Let $\mathcal{N}$ denote a finite set of smart objects (i.e. wireless devices referred as nodes) and let $n \in \mathcal{N}$ denote a single node. There is a special node that serves as the gateway (denoted by $n_{\mathrm{gw}}$ ) and the remaining nodes are divided into two types according their mobility:

- 1) stationary nodes (denoted by $\mathcal{N}_{\text {sn }}$ ) which are motionless after deployment (e.g. fixed to environments);
- 2) mobile nodes (denoted by $\mathcal{N}_{\mathrm{mn}}$ ) which can change their positions over time (e.g. deployed on animals).

A self-organised network can be constructed with these nodes without relying on additional communication infrastructures. Given a constant transmission radius (denoted by $R$ ) for each node, network connectivity depends on node positions as defined by a unit disk model [25].

Consider a package from the gateway $n_{\mathrm{gw}}$ to a group of target nodes (denoted by $\mathcal{N}_{\text {tgt }}$ ). To make the problem more tractable, we narrow down our research to the case where only mobile nodes are the package target, i.e. $\mathcal{N}_{\text {tgt }} \subset \mathcal{N}_{\text {mn }}$.

Let $\mathcal{N}_{\text {rly }}^{c}$ denote a set of candidate relay nodes. To avoid the costly maintenance of topological dynamics caused by node mobility, the relay candidates are limited to stationary nodes, i.e. $\mathcal{N}_{\text {rly }}^{\mathrm{c}} \subset \mathcal{N}_{\text {sn }}$. It is assumed that the delivery can be accomplished when the target node is directly in contact range of the selected relay(s).

To reach candidate relay nodes from the gateway, hopby-hop forwarding is required. The topology knowledge of stationary nodes is assumed to be available at the gateway $n_{\mathrm{gw}}$, as the positions of stationary nodes are certain once deployed. A hop distance function $f_{\text {hd }}\left(n_{i}\right)$ denotes the minimum number of forwarding hops from the gateway to any stationary node $n_{i} \in \mathcal{N}_{\text {sn }}$.

\section{Delay Budget}

Traditionally, the delivery is simply required to be finished before a maximum acceptable delay in previous works such as [11]. However, for many package delivery scenarios, there exists more-or-less flexibility regarding acceptable delay, which brings optimisation opportunities that are overlooked by existing works. To this end, our research describes the delay sensitivity by a two-level budget model:

- The first-level budget (called the main budget) has a soft boundary and any earlier delivery is considered to be a fully satisfied delivery.

- The second-level budget (called the extra budget) has a firm boundary and any later delivery is considered to be a fully unsatisfactory delivery.

- For the delivery delay between these two boundaries (i.e. within the range of the extra budget), it contributes to decreasing satisfaction of delivery.

This two-level budget model describes the relationship between delivery satisfaction and delivery delay, called a delay budget function. Although factors such as buffer limitation can be considered in the budget modelling (e.g. a stricter budget results from more limited storage), this paper assumes that the budget function is only related to the message delay tolerance, as our research focus is on investigating the utilisation of directional movement. In other word, all relay candidates are considered to have sufficient resource to support the delaytolerant relaying mechanism.

Theoretically, the delay budget function can be any type of monotonically decreasing function. In this paper, we focus on the sigmoid curve (a special case of the logistic function [26]) which allows smooth transition to reflect a realistic tolerance 
of acceptable delay. Thus, the raw delay budget function is given as

$$
f_{\mathrm{db}}^{*}(\tau)=\frac{1}{1+e^{\mu\left(\tau-\frac{\tau_{1}+\tau_{2}}{2}\right)}}
$$

where $\tau \geqslant 0$ denotes the delivery delay ${ }^{3}, \tau_{1}>0$ denotes the first-level budget boundary, $\tau_{2}>\tau_{1}$ denotes the second-level budget boundary ${ }^{4}$, and $\mu$ denotes a functional factor.

Given $\tau_{1}$ and $\tau_{2}$, the functional factor $\mu$ determines the curve shape. To calculate the value of $\mu$, we consider $f_{\mathrm{db}}^{*}\left(\tau_{1}\right)=$ $1-\epsilon$, where $\epsilon \in(0,1)$. A smaller $\epsilon$ brings more accuracy (because the budget function can have fewer decrements before $\tau_{1}$ ) and $\epsilon$ is set to be 0.001 which is sufficiently accurate for our research. Then the functional factor $\mu$ can be calculated as

$$
\mu=\frac{2 \ln \left((1-\epsilon)^{-1}-1\right)}{\tau_{1}-\tau_{2}} .
$$

Let the delivery satisfaction be a degree value between 0 and 1 , so the budget function is further normalised as

$$
f_{\mathrm{db}}(\tau)=\frac{1+e^{-\mu\left(\frac{\tau_{1}+\tau_{2}}{2}\right)}}{1+e^{\mu\left(\tau-\frac{\tau_{1}+\tau_{2}}{2}\right)}}
$$

In Figure 2, the delay budget function is illustrated for the example where the main budget is 500 seconds. To keep this figure concise, only one case of extra budget is indicated (which can be shown similarly for the other two example cases). This figure shows that the delivery satisfaction starts to decrease from the first-level budget boundary and becomes zero at the second-level budget boundary. With such a transition range, a larger delivery delay is still allowed (but less encouraged) even if the main budget is exceeded, which provides more flexibility in relay selection. For instance, the possibility of 510 seconds delay can be considered for making a possible better offer in other respects (e.g. much fewer forwarding hops). The optimisation opportunity brought by this flexible delivery delay requirement will be further investigated in the remainder of this paper.

\section{Contact Opportunity}

An analytical model referred from [12] is used to describe the contact opportunity between mobile target and stationary relay. This contact model is derived from an analysis of correlated random walks, which reflects the opportunistic contact caused by random movements with directional correlation.

Based on the random walk mobility model [27], the mobile target is assumed to take successive steps (each step has a constant length denoted by $L=V \cdot T$, where $V$ denotes an average movement speed and $T$ denotes a constant step period) and takes a random turn after each step. The random turning angle between two successive steps is assumed to follow the zero-centred Normal distribution, where the Standard Deviation of the Turning Angles (SDTA) is denoted by $\sigma$. When the

\footnotetext{
${ }^{3}$ As the delivery delay of FWD strategy is dominated by the waiting time at the stationary relay, the transmission delay is considered to be negligible in our research, and the case of zero delay represents direct delivery without waiting.

${ }^{4}$ When the two budget boundaries are close enough $\left(\left(\tau_{2}-\tau_{1}\right)<0.001\right.$ for this paper), it is treated as no extra budget as mentioned in Section V.
}

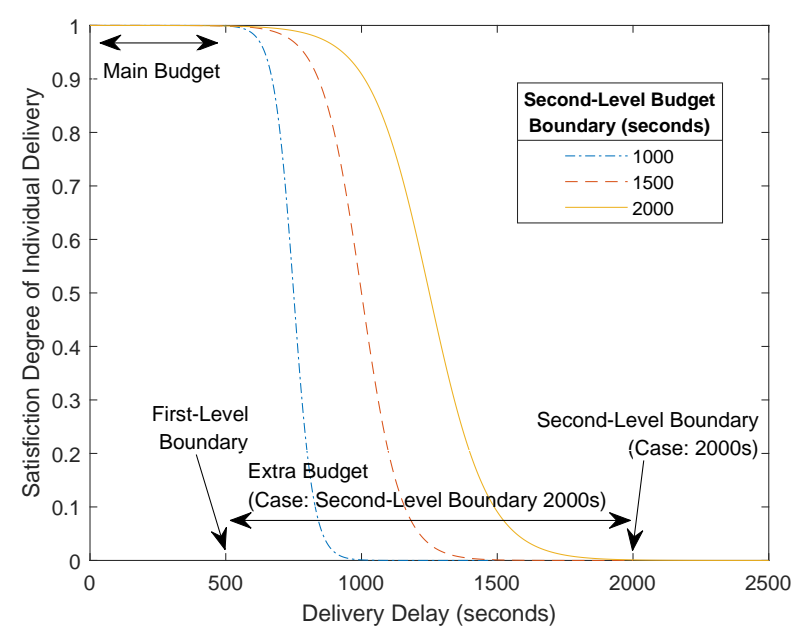

Fig. 2. Delay budget function when the main budget is 500 seconds

directional correlation is strong (i.e. $\sigma$ is small), the mobile target tends to keep moving in the current direction, which results in a directional movement.

The contact certainty between a mobile target and a stationary relay is formulated on the basis of a geometric relationship. As shown in Figure 3, their relative position is the key modelling aspect. Given the relative distance (denoted by $r$ ) from the original position of the mobile target, the minimum required number of steps is calculated as $\lambda=\frac{r-R}{L}(\lambda=0$ if $r \leqslant R$ ). Given a relative angle (denoted by $\varphi$ ) measured with respect to the initial movement direction of the mobile target, a range of directional coverage is calculated as

$$
\left\{\begin{array}{l}
\varphi_{\min }^{\mathrm{cover}}=\varphi-\sin \left(\frac{R}{r}\right) \\
\varphi_{\max }^{\operatorname{cover}}=\varphi+\sin \left(\frac{R}{r}\right)
\end{array}\right.
$$

where $\varphi_{\min }^{\text {cover }}, \varphi_{\max }^{\text {cover }} \in[-\pi, \pi]$ denotes the minimum/maximum coverage angle and $R$ denotes the radius of contact range.

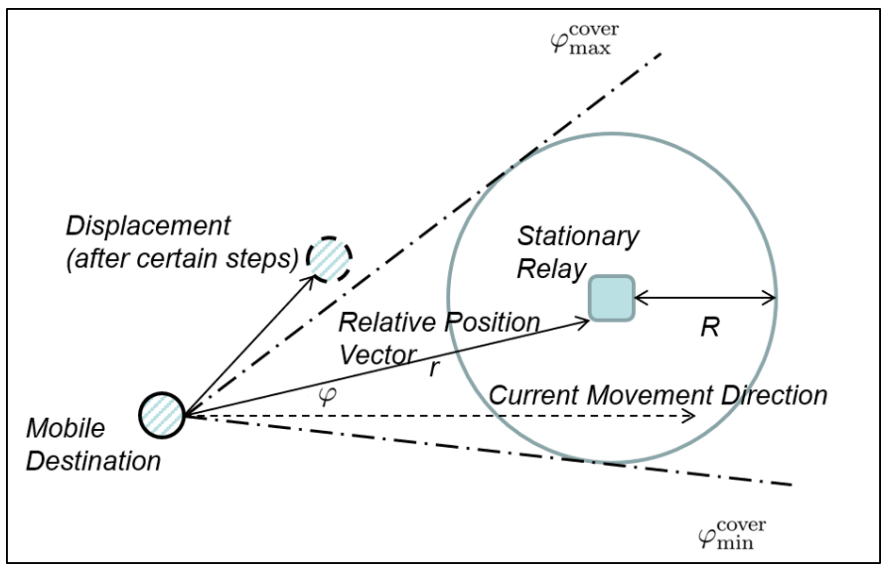

Fig. 3. Illustration of the geometric relationship for contact modelling

Finally, the contact certainty function can be given as

$$
f_{\mathrm{cc}}^{(\sigma)}(r, \varphi)=\int_{\varphi_{\min }^{\text {cover }}}^{\varphi_{\max }^{\text {cover }}} f_{\mathrm{pdf}}(\delta, \sigma, \lambda) d \delta
$$


where $f_{p d f}$ denotes the parametric function obtained from simulations referring [12], which reflects the probability distribution of displacement angle after $\lambda$ steps given certain $\sigma$.

\section{Relay StRategy For EFFICIENT GROUP DELIVERY}

Given the underlying schemes that are already described in Section III, the research problem now focuses on the optimisation of the relay strategy. The relay strategy is considered to be a decision (made by the gateway) that determines relay selection and target assignment for achieving a group delivery.

Different from individual delivery, relay nodes can be shared by multiple target nodes, and therefore more optimisation is possible. Based on knowledge of predictable node contact and tolerable delivery delay, how to identify feasible opportunities for relay sharing is the key issue to be addressed. In this section, the research problem is formulated and solutions are proposed.

\section{A. Problem Formulation}

Two optimisation objectives are considered to formulate the research problem: 1) maximise the cost reduction of group delivery; 2) maximise the requirement satisfaction of group delivery. To reflect potential conflicts between these two objectives, this optimisation problem is required to be formally formulated for further analysis.

Let $x_{i j}$ denote a binary indicator of the target assignment decision, such that

$$
x_{i j}=\left\{\begin{array}{cc}
1 & \text { if relay } n_{i} \text { is responsible for target } n_{j} \\
0 & \text { otherwise }
\end{array}\right.
$$

where $n_{i} \in \mathcal{N}_{\text {rly }}^{\mathrm{c}}$ denotes a candidate relay node and $n_{j} \in \mathcal{N}_{\text {tgt }}$ denotes a target node. For the sake of simplicity, it is assumed that $\mathcal{N}_{\text {rly }}^{\mathrm{c}}=\mathcal{N}_{\text {sn }}$ and $\mathcal{N}_{\text {tgt }}=\mathcal{N}_{\text {mn }}$ in the remainder of this paper.

Then a relay strategy matrix (denoted by $X$ ) can be represented as

$$
X=\left[\begin{array}{ccccc}
x_{11} & x_{12} & x_{13} & \ldots & x_{1 J} \\
x_{21} & x_{22} & x_{23} & \ldots & x_{2 J} \\
\vdots & \vdots & \vdots & \ddots & \vdots \\
x_{I 1} & x_{I 2} & x_{I 3} & \ldots & x_{I J}
\end{array}\right]=\left(x_{i j}\right)_{I \times J}
$$

where $I$ denotes the number of candidate relay nodes, and $J$ denotes the number of delivery target nodes.

For the strategy matrix $X$, each row represents the relay node selection for a specific target node $n_{j}$ and each column represents the target node assignment for a specific candidate relay node $n_{i}$. The whole set of selected relays can be represented as

$$
\mathcal{N}_{\text {rly }}=\left\{n_{i} \mid x_{i j}=1\right\}, n_{j} \in \mathcal{N}_{\text {tgt }}
$$

Note that multiple target nodes may have the same relay node, which is equivalent to sharing a relay node for a subgroup of targets. The subgroup of targets which are assigned to $n_{i}$ can be represented as

$$
\mathcal{N}_{\text {tgt } \mid n_{i}}=\left\{n_{j} \mid x_{i j}=1\right\}, n_{i} \in \mathcal{N}_{\text {rly }}^{\mathrm{c}}
$$

Referring Section III-D, let $\vec{\rho}_{i j}=\left(r_{i j}, \varphi_{i j}\right)$ denote the relative position between stationary node $n_{i}$ and mobile node $n_{j}$. The contact delay between $n_{i}$ and $n_{j}$ (denoted by $\tau_{i j}$ ) can be estimated given the minimum required steps so that $\tau_{i j}=\lambda \cdot T$. For each $n_{j} \in \mathcal{N}_{\text {tgt }}$, its best selected relay is denoted as $n_{*}=\arg \max _{n_{i} \in \mathcal{N}_{\text {rly }}}\left(f_{\mathrm{cc}}\left(\vec{\rho}_{i j}\right) \cdot f_{\mathrm{db}}\left(\tau_{i j}\right)\right)$, subject to $n_{j} \in \mathcal{N}_{\text {tgt } \mid n_{i}}$. Pairing $n_{j}$ with its best selected relay, $\vec{\rho}_{* j}$ denotes their relative position and $\tau_{* j}$ denotes their estimated contact delay.

Under the relay strategy $X$, let $C_{X}^{*}$ denote the overall reduction $^{5}$ of forwarding hops and $D_{X}^{*}$ denote the overall satisfaction $^{6}$ of delivery requirement, i.e.

$$
\begin{gathered}
C_{X}^{*}=H-\sum_{n_{i} \in \mathcal{N}_{\mathrm{rly}}} f_{\mathrm{hd}}\left(n_{i}\right) \\
D_{X}^{*}=\sum_{n_{j} \in \mathcal{N}_{\mathrm{tgt}}}\left(f_{\mathrm{cc}}\left(\vec{\rho}_{* j}\right) \cdot f_{\mathrm{db}}\left(\tau_{* j}\right)\right)
\end{gathered}
$$

where $H$ denotes the sum of least forwarding hops for group relay selection based on immediate delivery (i.e. no movement prediction).

Then two optimisation objectives are defined as

$$
\begin{gathered}
f_{1}^{\mathrm{obj}}(X)=\frac{H-\sum_{n_{i} \in \mathcal{N}_{\mathrm{rly}}} f_{\mathrm{hd}}\left(n_{i}\right)}{H}=C_{X} \\
f_{2}^{\mathrm{obj}}(X)=\frac{\sum_{n_{j} \in \mathcal{N}_{\mathrm{tgt}}}\left(f_{\mathrm{cc}}\left(\vec{\rho}_{* j}\right) \cdot f_{\mathrm{db}}\left(\tau_{* j}\right)\right)}{J}=D_{X}
\end{gathered}
$$

where $J$ denotes the number of targets, $C_{X}$ is called the reduction degree of overall cost (the objective denoted by the function $f_{1}^{\mathrm{obj}}$ ), and $D_{X}$ is called the satisfaction degree of group delivery (the objective denoted by the function $f_{2}^{\text {obj }}$ ).

The optimisation problem can finally be formulated as

$$
\max _{X \in \Omega}\left(f^{\mathrm{obj}}(X)\right)
$$

subject to

$$
\begin{gathered}
\left|\mathcal{N}_{\text {rly }}\right| \leqslant J \\
\sum_{n_{i} \in \mathcal{N}_{\text {rly }}} f_{\text {hd }}\left(n_{i}\right)<H
\end{gathered}
$$

where $\Omega$ denotes the feasible strategies under the subject condition, and $f^{\text {obj }}(X)=\left[f_{1}^{\text {obj }}(X), f_{2}^{\text {obj }}(X)\right]$ denotes the vector of two objectives. Equation (15) is the constraint that the number of selected relays (denoted by $\left|\mathcal{N}_{\text {rly }}\right|$ ) should be no more than the number of targets. Equation (16) is the constraint that the sum of forwarding hops should be less than employing the strategy of immediate delivery.

Referring [11], the formulated problem for efficient group delivery is NP-hard. To solve this problem, algorithms are designed and evaluated in the remainder of this paper. With the consideration of a two-level delay budget model in biobjective combinatorial optimisation, our study is distinct from the existing research.

\footnotetext{
${ }^{5}$ Multicast tree construction is not considered in this paper but further research can be conducted from this basic case.

${ }^{6}$ It is assumed that each individual delivery independently contributes to the group delivery, so the overall satisfaction is a sum of individual satisfaction.
} 


\section{B. Group Relay Selection (Evolutionary Approach)}

As a popular approach to solving multi-objective optimisation problems, evolutionary algorithms can produce a number of potential solutions for making further decisions. When these solutions are Pareto optimal (i.e. an improvement in any objective requires a degradation in other objective(s)), a Pareto front (i.e. the set of all Pareto optimal solutions) can be approximated to analyse the trade-off between objectives [28].

In our research, the Multi-Objective Genetic Algorithm (MOGA) [29] is adopted because it is a standard evolutionary algorithm which is suitable for solving combinatorial optimisation problems. Based on the MOGA implementation ${ }^{7}$, the group relay selection is considered from following three aspects:

1) Population: The main issue to be addressed in the evolutionary approach design is to reduce the solution space for better and faster searching. Without such reduction, it is found hard to obtain a suitable solution in limited time due to the existence of too many possibilities.

Therefore, instead of using the strategy matrix $X$, a chromosome is proposed to be a binary selection vector (denoted by $\vec{z}$ ) as

$$
\vec{z}=\left[\begin{array}{c}
z_{1} \\
z_{2} \\
\vdots \\
z_{I}
\end{array}\right]=\left(z_{i}\right)_{I}
$$

Then a combination of selected relays can be indicated by

$$
\mathcal{N}_{\text {rly }}=\left\{n_{i} \mid z_{i}=1\right\}, n_{i} \in \mathcal{N}_{\text {rly }}^{\mathrm{c}}
$$

Let $\mathcal{Z}$ denote a population which consists of a number of chromosomes. An initial population (generation zero) is generated randomly ${ }^{8}$ as

$$
\mathcal{Z}^{(0)}=\left\{\vec{z}_{1}, \vec{z}_{2}, \ldots, \vec{z}_{K}\right\}
$$

where $K$ denotes the number of chromosomes in the population.

2) Calculation: Although the searching space can be reduced by using the selection vector $\vec{z}$, a strategy matrix is still required to evaluate the chromosome fitness (i.e. the two optimisation objectives). For generating the strategy matrix from a selection vector, a default assignment matrix is defined as $\hat{X}=\left(\hat{x}_{i j}\right)_{I \times J}$ such that,

$$
\hat{x}_{i j}=\left\{\begin{array}{cc}
1 & \text { if } f_{\mathrm{cc}}\left(\vec{\rho}_{i j}\right) \cdot f_{\mathrm{db}}\left(\tau_{i j}\right)>0 \\
0 & \text { otherwise. }
\end{array}\right.
$$

which means that each target node is assigned to all possible relay candidates.

Given a selection vector $\vec{z}$, corresponding raw strategy matrix (denoted by $X^{*}$ ) based on the default assignment can be generated as

$$
X^{*}=\left(x_{i j}^{*}\right)_{I \times J}=\vec{z} \cdot \hat{X}
$$

\footnotetext{
${ }^{7}$ A controlled, elitist genetic algorithm (a variant of NSGA-II [29]) provided by the MATLAB Global Optimization Toolbox. The algorithm follows default settings of this standard implementation unless otherwise stated.

${ }^{8}$ For Section V-D, the solutions found by four greedy schemes are included in the initial population to ensure the searching performance.
}

Note that the raw strategy matrix brings redundant assignments (i.e. a target node is assigned to more than one relays). Although this redundancy can improve the delivery reliability, our research focuses on the analysis of the relay sharing opportunity to reduce the delivery cost and therefore a basic case is considered that one target is only assigned to one relay (but further research can be conducted from this basic case). Based on Algorithm 1, the raw strategy matrix is adjusted to eliminate the redundancy and then corresponding fitness values can be calculated.

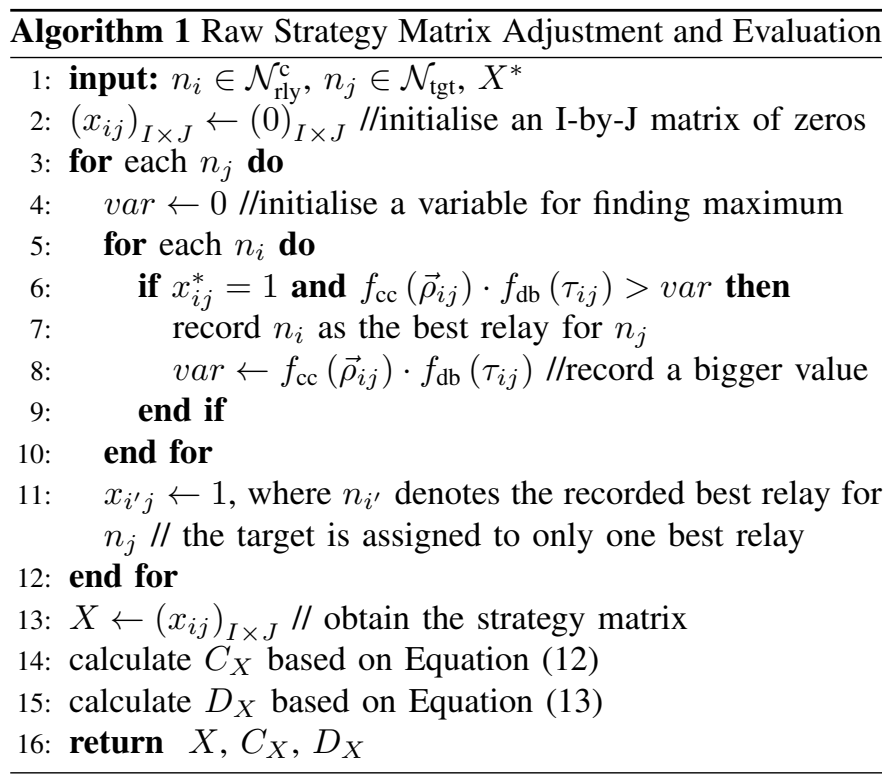

3) Iteration: Given an initial population and the specified fitness calculation, the algorithm leads later generations to evolve towards the optimal solutions iteratively. The production of a new generation follows a general procedure as:

- select parents from the current population;

- create children by using genetic operators (crossover and mutation) on the selected parents;

- calculate fitness using the Algorithm 1 and eliminate inferior chromosomes to retain a fixed size of population.

The iterative mechanism finally terminates when the process reaches a specified generation/time limit or there is sufficient convergence in the solutions. A set of Pareto optimal solutions is provided when the algorithm terminates along with their achieved fitness values.

\section{Group Relay Selection (Greedy Approach)}

Because evolutionary algorithms always require iterative computation which can be inefficient, the relay selection based on a greedy algorithm is proposed as an alternative. The principle of a greedy algorithm is choosing the best solution for each sub-problem until the whole problem can be solved [22]. It is a straightforward approach which is fast and simple especially when solving complex problems. Despite its simplicity, the performance of a greedy algorithm should be carefully evaluated because greedy searching towards a local optimum may not lead to global optimisation. 
In [11], a greedy algorithm is proposed for trajectory-based group message delivery. With the relay selection performed by this algorithm, relay sharing opportunities can be exploited for group delivery so that the overall forwarding hops can be reduced for a given delay budget. Specifically, the problem of group relay selection is solved in two phases (as shown in Algorithm 2):

- For the first phase, the serving list is identified as a list of targets who can be served by a certain stationary relay.

- For the second phase, one stationary relay is selected each round until the serving lists of the selected relays can cover all mobile targets.

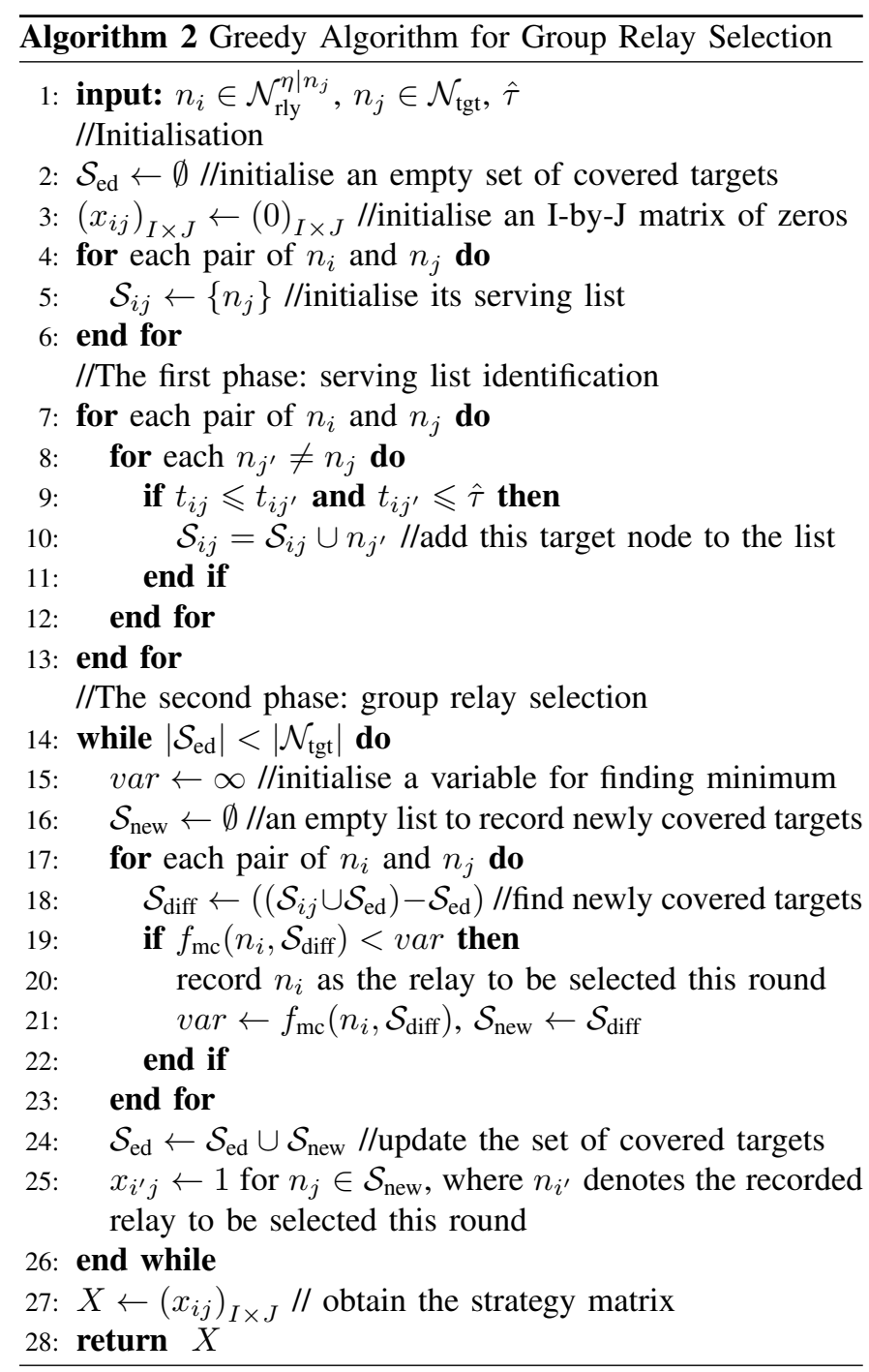

However, it is highlighted that the algorithm in [11] is not directly applicable to our research scenario in regard to the following two aspects:

- Firstly, accurate trajectory knowledge, assumed by [11], is unavailable so the serving list cannot be explicitly known.

- Secondly, an exact budget boundary may not exist due to the existence of the extra budget. This situation is not considered by [11].
In order to achieve greedy relay selection based on directional movement with awareness of extra budget, we decouple the problem solving into two complementary steps:

- The method of Delay Boundary based Relay Selection (DBRS) is proposed for the scenario where mobile nodes randomly move with directional correlation. The DBRS is considered as the performance benchmark because it has no awareness of the extra delay budget as with the method proposed in [11].

- A new method of Satisfaction Degree based Relay Selection (SDRS) is proposed to consider the extra delay budget in group relay selection. Based on the design of DBRS, the SDRS has additional awareness so better performance can be expected.

1) DBRS Design: The main issue to be addressed in the DBRS design is the identification of the serving list in the first phase of the problem solution. Without accurate trajectory knowledge, it becomes hard to identify a list of targets who can be served by a certain stationary relay.

To overcome the difficulty brought about by the randomness of node movement, a threshold (denoted by $\eta$ ) of contact certainty is proposed to qualify a set of candidate stationary relays as

$$
\mathcal{N}_{\text {rly }}^{\eta \mid n_{j}}=\left\{n_{i} \mid f_{\text {cc }}\left(\vec{\rho}_{i j}\right) \geqslant \eta\right\}, n_{i} \in \mathcal{N}_{\text {rly }}^{\mathrm{c}}
$$

With the qualified candidates, a serving list (denoted by $\mathcal{S}_{i j}$ ) can be found for each pair of Stationary Relay $n_{i}$ and Mobile Target $n_{j}$ (called the SRMT pair) according to the condition that

$$
\mathcal{S}_{i j}=\left\{n_{j^{\prime}} \mid \tau_{i j} \leqslant \tau_{i j^{\prime}} \leqslant \hat{\tau}\right\} n_{j} \in \mathcal{N}_{\mathrm{tgt}}, n_{j^{\prime}} \in \mathcal{N}_{\mathrm{tgt}}
$$

where $\hat{\tau}$ denotes a budget boundary to identify a serving list.

Then, the second phase of problem solution starts from an empty set of covered targets. After each evaluation round, one stationary relay will be selected and the number of covered targets will increase (added from corresponding serving list). The procedure of relay selection ends when the selected relays can cover all mobile targets.

Given the current set of covered targets, each SRMT pair can be evaluated by a cost metric (the hop distance averaged by the number of newly covered targets). The calculation of this cost metric is same as the cost calculation proposed by [11] as

$$
f_{\mathrm{mc}}^{\mathrm{DB}}\left(n_{i}, \mathcal{S}_{\mathrm{diff}}\right)=\frac{f_{\mathrm{hd}}\left(n_{i}\right)}{\left|\mathcal{S}_{\mathrm{diff}}\right|}
$$

where $f_{\mathrm{mc}}^{\mathrm{DB}}$ denotes the metric calculation used in DBRS, $f_{\mathrm{hd}}$ denotes the hop distance function (hops from the gateway) defined in Section III-B, $\mathcal{S}_{\text {diff }}$ denotes a list of newly covered targets (obtained from line 18 in Algorithm 2), and $\left|\mathcal{S}_{\text {diff }}\right|$ denotes the size of this list.

Note that this DBRS scheme relies on the assumption that all deliveries before a given delay $\hat{\tau}$ contribute the same degree of delivery satisfaction. However, in our research scenario, the satisfaction degree gradually decreases after the first-level budget boundary is exceeded. Without this awareness in the algorithm, optimisation opportunities can be overlooked and therefore the SDRS design is proposed. 
2) SDRS Design: The main issue to be addressed in the SDRS design is the consideration of the extra delay budget in the second phase of the problem solution. During the first phase, it is similar to our DBRS design except that the budget boundary is fixed to the second-level budget boundary (i.e. $\hat{\tau}=\tau_{2}$ ) to provide all possible candidate stationary relays.

After the first phase, all the contactable targets within the second-level budget boundary are included in the serving list. Thus the cost metric should reflect the difference in delivery satisfaction. Instead of treating all the newly served targets the same, the hop distance is averaged by the sum of their satisfaction degree as

$$
f_{\mathrm{mc}}^{\mathrm{SD}}\left(n_{i}, \mathcal{S}_{\mathrm{diff}}\right)=\frac{f_{\mathrm{hd}}\left(n_{i}\right)}{\sum_{n_{j} \in \mathcal{S}_{\mathrm{diff}}} f_{\mathrm{db}}\left(\tau_{i j}\right)}
$$

where $f_{\mathrm{mc}}^{\mathrm{SD}}$ denotes the metric calculation used in SDRS and $f_{\mathrm{db}}$ denotes the delay budget function defined in Section III-C.

Compared with DBRS, a more generalised form of cost calculation is provided by SDRS. Because the delivery satisfaction is considered in relay selection, SDRS can make improved decisions and therefore better delivery performance can be achieved. In the next section, DBRS and SDRS will be compared and evaluated, together with the evolutionary approach proposed in Section IV-B.

\section{Simulation and Evaluation}

In Section IV, the research problem is formulated and solutions are proposed to select relay node(s) for group delivery. Now, we further evaluate the performance of proposed solutions in simulation scenarios.

\section{A. Scenario Description}

Figure 4 shows a scenario where a number of stationary nodes (denoted by squares) are deployed over a geographical area. Without loss of generality, a base scenario is defined for our simulations that stationary nodes are uniformly distributed over $10000 * 10000$ metre area as a $21 * 21$ grid. At certain time point during network operations (treated as the current time), a package is planned to be delivered to a group of mobile nodes (denoted by circles) which are randomly located within the base scenario area. For the sake of simplicity, it is assumed that the package source (i.e. the gateway) is the stationary node at the bottom right corner. Although a group can include any number of targets, we focuses on a moderate size of the target group (around 20 mobile nodes) because neither unicast and broadcast is efficient in such range.

In our research, the radio coverage is considered to be limited (e.g. subject to device size and/or surrounding environment such as forest or underwater) and therefore the package delivery mainly relies on multi-hop forwarding. It is assumed that the stationary nodes can form a grid topology to perform forwarding. Considering the base scenario used for our simulations, the communication radius of wireless nodes is set to be $750 \mathrm{~m}$ (which is a feasible range referring [30]) so that each stationary node is able to contact its neighbour nodes.

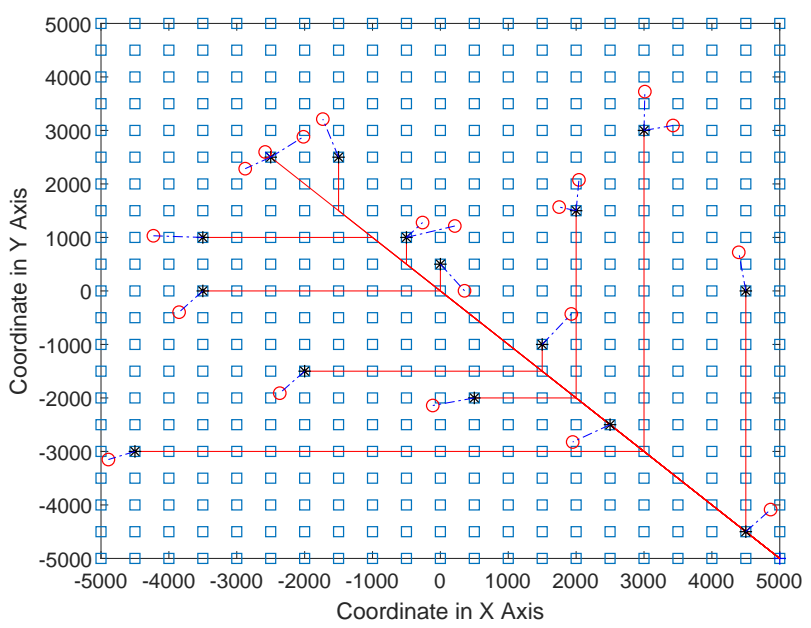

Fig. 4. Example simulation scenario (relay sharing without movement prediction)

With the FWD strategy (referring Section II-A), it is typically possible to achieve group package delivery by fewer forwarding hops. Instead of immediate delivery to each target, the package can be forwarded (via routes shown as solid lines) to the selected relay nodes (denoted by asterisks) and wait to be delivered when the target comes within the relay node coverage (indicated by dash-dot lines). By exploiting predictable contact and delay budget, the FWD strategy brings the opportunity of relay sharing to further reduce the overall forwarding hops. For the instance shown in Figure 5, only ten relay nodes are used for twenty targets (instead of fifteen relay nodes used in Figure 4) and therefore the overall forwarding hops can be reduced, by exploiting movement prediction in relay selection.

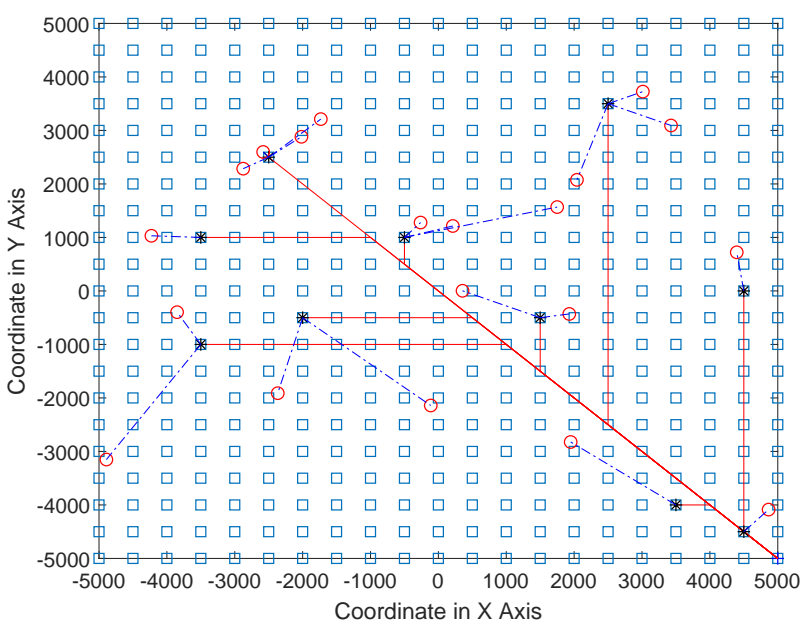

Fig. 5. Example simulation scenario (relay sharing with movement prediction)

Due to the contact uncertainty caused by movement randomness, proper selection of relay nodes is necessary to ensure delivery satisfaction. For the example simulation scenario (as shown in Figure 4 and 5), the trade-off between two objectives 
can be reflected by the plot of the Pareto front (as shown in Figure 6) which is obtained by MOGA (i.e. the evolutionary approach). Figure 6 shows that the cost reduction can firstly be improved without much loss of delivery satisfaction but then the delivery satisfaction will degrade rapidly with little further increase in the cost reduction. This means a further reduction of the delivery cost (beyond a certain point) notably impacts on the delivery satisfaction and the two objectives are then indeed conflicted.

To further evaluate the overall delivery performance, the weighted sum of two objectives is calculated and shown in Figure 7. For an easier understanding, it is worth noting that Figure 7 transforms the two objectives into one weighted sum value. So the two-dimensional plot shown by Figure 6 becomes multiple one-dimensional (vertically aligned asterisks) plots in Figure 7, given different weights of the cost reduction (the weight of the delivery satisfaction can be known at the same time as the sum of weights given as 1). With different weight values of the cost reduction, Figure 7 shows the limit of overall delivery performance is a curve and there is a concave region around the value where two weights are similar. This concave region indicates the two objectives cannot be simultaneously achieved, which results in a performance limit. It is also shown that the concave region is biased to the higher weight for cost reduction, which means it is harder to achieve cost reduction than the delivery satisfaction.

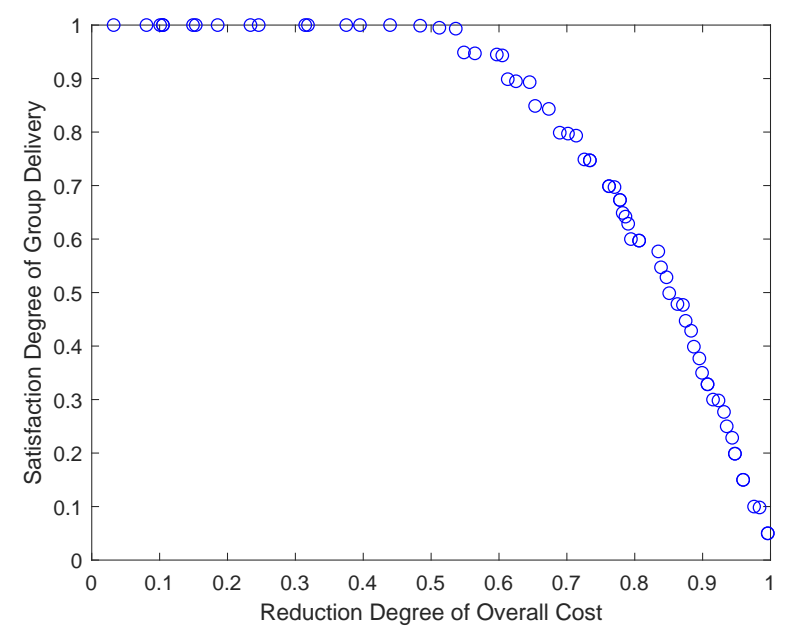

Fig. 6. Trade-off between cost reduction and delivery satisfaction shown by Pareto front for the example simulation scenario (main budget is 2000s without extra budget)

\section{B. Simulation Settings}

To evaluate the delivery performance, simulation results are obtained from random scenarios. As described in Section V-A, the scenario randomness comes from the mobile targets (i.e. the initial location and direction are randomly chosen for each simulation run). The obtained results are the average of multiple simulation runs (50 random seeds used).

By considering two optimisation objectives as metrics, this part investigates how the simulation is influenced by three re-

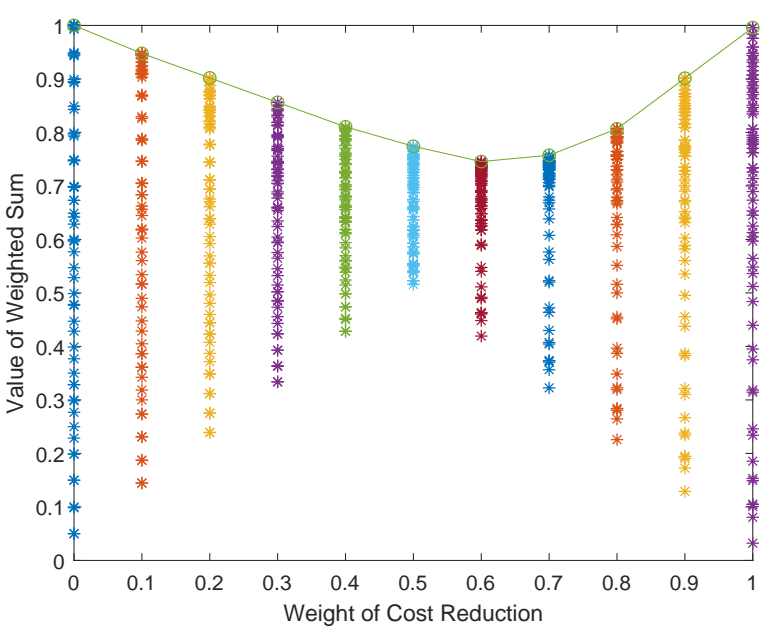

Fig. 7. Weighted sum of Pareto front over different weight values

lated factors, including the certainty threshold $\eta$, the Standard Deviation of the Turning Angles (SDTA) $\sigma$, and the target node number $J$. The extra budget is set to 0 for this part so there is no difference between DBRS and SDRS. The main budget is the control variable and the default value for other settings are $\eta=0.9, \sigma=5$, and $J=20$ (obtained from our investigations).

1) Certainty Threshold: With different values of certainty threshold, Figure 8 shows that a lower threshold does not improve the cost reduction much but sightly degrades the delivery satisfaction. Therefore the default value of certainty threshold is set to 0.9 for the remaining simulations unless otherwise stated.
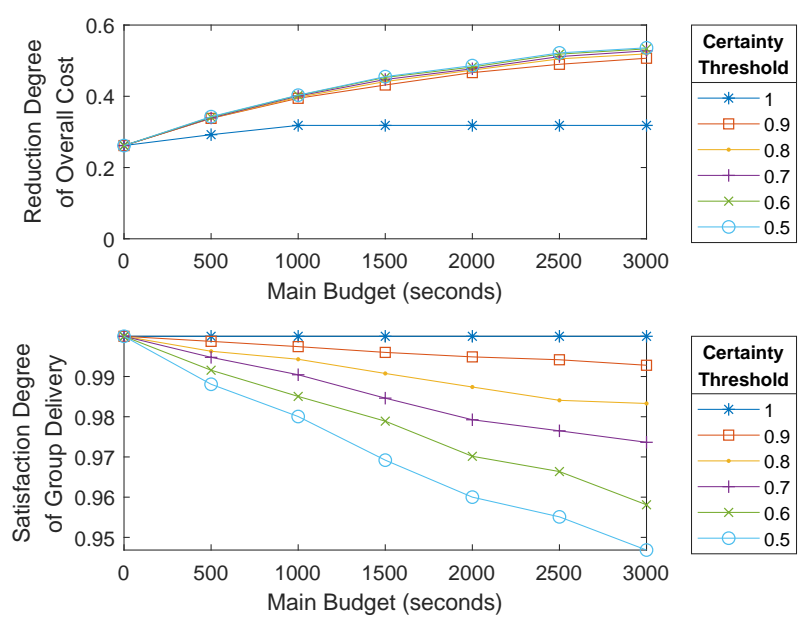

Fig. 8. Investigation of certainty threshold (no extra budget)

2) Target Node Number: With different values of main budget, Figure 9 shows that the cost reduction increases with target number. Given the number of target nodes, higher reduction degree can be achieved by a larger delay budget, because there are more opportunities of relay sharing. The satisfaction degree is relatively steady but reflects a similar 
trend, due to the trade-off between the cost reduction and delivery satisfaction. As our research focus is on the group delivery, a relative large group is considered and the default value of target number is set to 20 for remaining simulations unless otherwise stated.
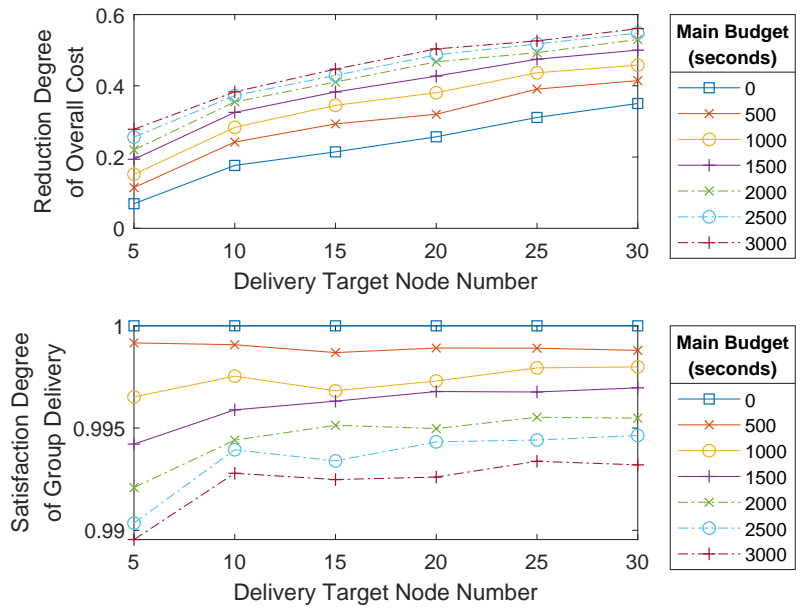

Fig. 9. Investigation of target node number (no extra budget)

3) SDTA: Figure 10 shows that different values of the main budget can lead to different delivery performance especially when SDTA is relatively small. With increasing SDTA, the influence of the main budget decreases as the movement is less predictable for exploitation. Meanwhile, after the decrease when the SDTA is set to certain value (e.g. 10 degrees in this case), the delivery satisfaction increases again and even surpasses the satisfaction when the SDTA is lower. This concave region shown in the delivery satisfaction is a result of less opportunistic forwarding (i.e. a stronger tendency to deliver immediately instead of waiting for later contact), when less contact opportunities can be identified by the prediction model (referring to Section III-D). As our research focuses on directional movement with strong correlations, a small SDTA is considered and the default value of SDTA is set to be 5 degrees for the remaining simulations unless otherwise stated.

\section{Performance Comparison of Greedy Schemes}

This section provides a performance comparison of relay selection based on different greedy schemes. Referring the delay budget model described in Section III, three kinds of budget boundary are considered for the DBRS: no delay budget (DBRS-N), first-level budget boundary (DBRS-F), and second-level budget boundary (DBRS-S). As mentioned in Section IV-C, SDRS is the proposed scheme with extra budget awareness and is highlighted for comparison focusing on the benefit of the extra budget term. As discussed in Section V-B, the following default settings are adopted for the factors: $\eta=0.9, \sigma=5$, and $J=20$.

1) Zero Main Budget: In Figure 11, the DBRS-N and DBRS-F schemes have the same performance as when the main budget is set to be zero. Furthermore, the performance of DBRS-N and DBRS-F do not vary with extra budget
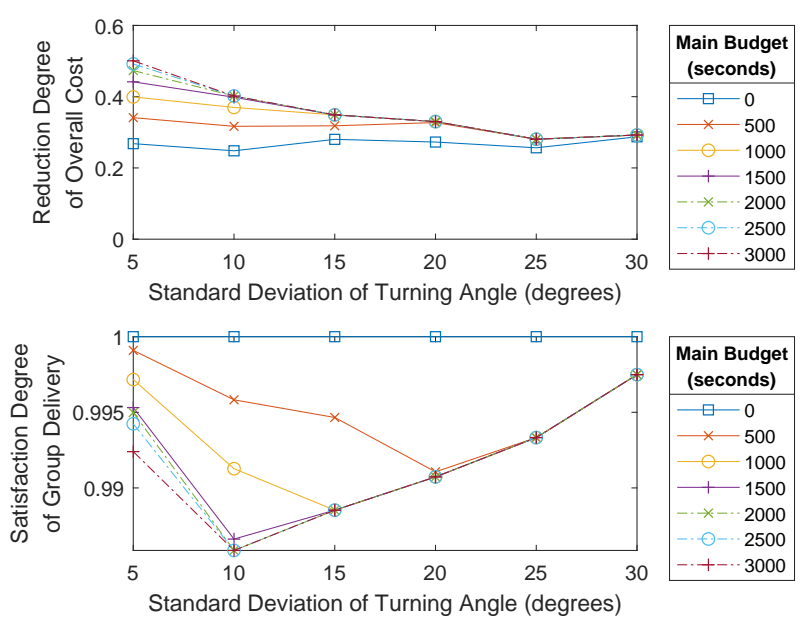

Fig. 10. Investigation of SDTA (no extra budget)

due to none awareness of this factor. With increasing value of extra budget, DBRS-S and SDRS both have increasing cost reduction degree and decreasing ${ }^{9}$ delivery satisfaction degree. Although more cost reduction can be achieved by DBRS-S, SDRS can have much less effect on the satisfaction given a similar cost reduction as the delay budget function is considered in relay selection.
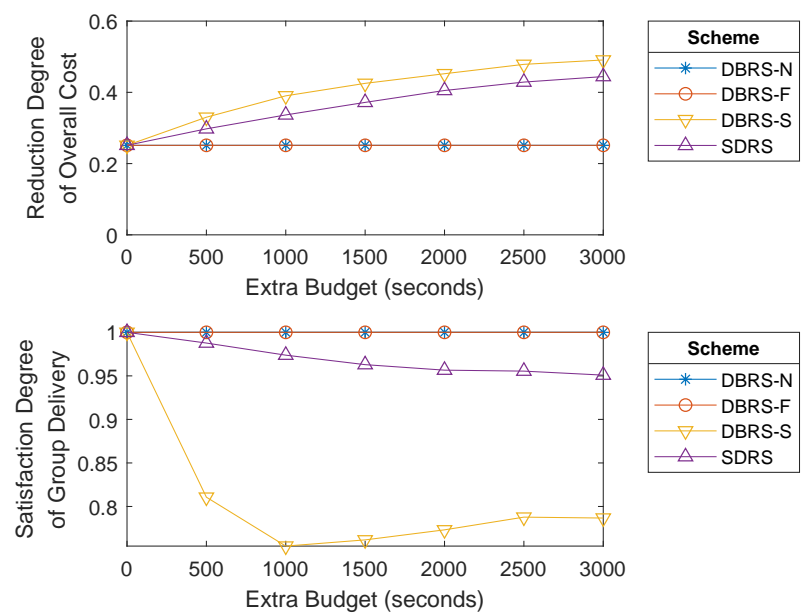

Fig. 11. Comparison of greedy schemes for zero main budget

2) Small Main Budget: For Figure 12, a small main budget is provided (set to 500 seconds). DBRS-F can achieve a higher cost reduction degree than DBRS-N because the main budget brings more relay candidates into consideration and more opportunity of relay sharing. Due to the strong directional

${ }^{9}$ For the DBRS-S, the delivery satisfaction degree slightly increases when the extra budget is larger than certain value. The reason is that the delay budget function (i.e. delivery requirements) is changed with the changing value of extra budget. Then the same delivery delay (if within the range of extra budget) contributes more delivery satisfaction and therefore the overall satisfaction is increased. Because the delivery delay tends to be larger with a higher value of main budget, this effect becomes even more significant in Figure 12, 13, 14, and SDRS is also affected in Figure 14. 
correlation reflected by the small SDTA in the simulation settings, a small main budget rarely introduces uncertainty and therefore the satisfaction of DBRS-F is almost the same as with DBRS-N. In addition, the performance of DBRS-N and DBRS-F do not vary with extra budget due to their lack of awareness of this factor. DBRS-S and SDRS are similar in terms of their performance in Figure 11, because a small value of main budget does not introduce much difference compared with a zero main budget.
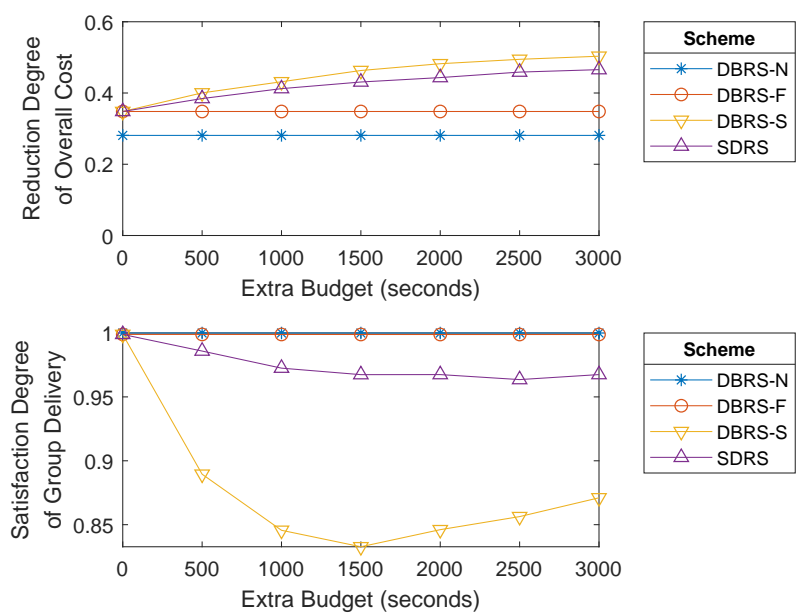

Fig. 12. Comparison of greedy schemes for small main budget (500s)

3) Medium Main Budget: For Figure 13, a larger main budget is introduced as 1000 seconds so the gap between DBRS$\mathrm{N}$ and DBRS-F (over the cost reduction degree) is increased. Meanwhile, the delivery satisfaction of DBRS-F shows a slight degradation because of the larger main budget brings more uncertainty. Although more cost reduction can still be achieved by DBRS-S and SDRS, the reduction gain brought about by the extra budget becomes smaller when a medium main budget is adopted, which reflects less opportunities for exploitation.
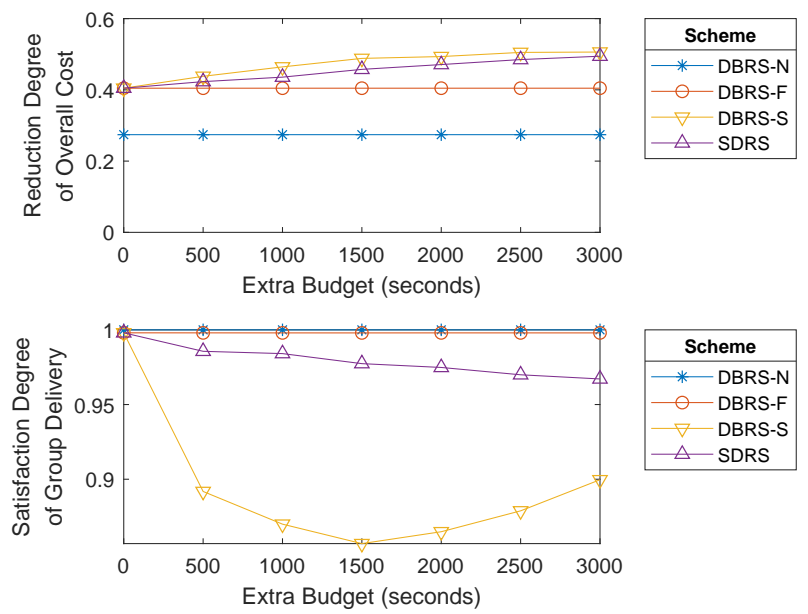

Fig. 13. Comparison of greedy schemes for medium main budget (1000s)
4) Large Main Budget: As shown in Figure 14, DBRSF can nearly achieve the same cost reduction as DBRS$\mathrm{S}$ and SDRS, when the main budget is much larger (2000 seconds in this case). However, this brings a more obvious difference between DBRS-N and DBRS-F in terms of delivery satisfaction. The extra budget can hardly bring additional benefit as the directional movement has been exploited by the very large main budget.
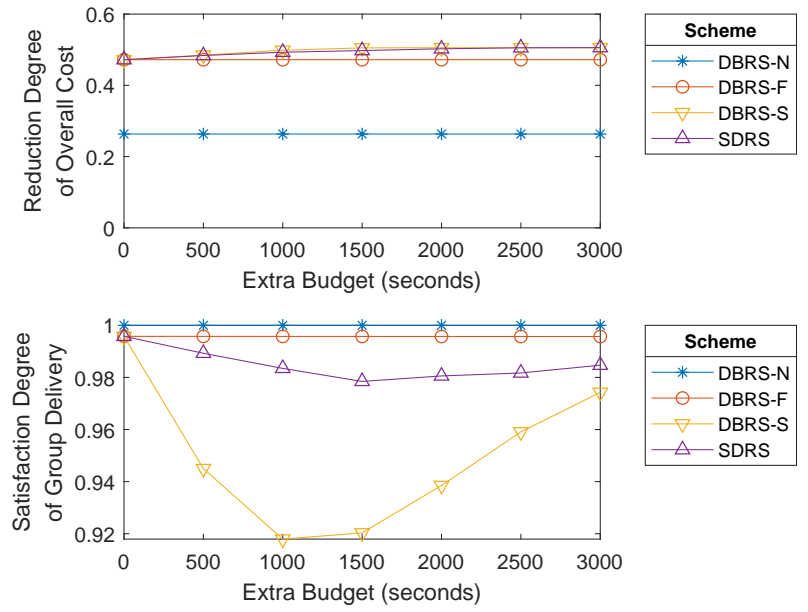

Fig. 14. Comparison of greedy schemes for large main budget (2000s)

5) Summary: Based on the above discussions, SDRS potentially brings efficiency gains at reasonable cost. Focused on the case of a small main budget, we further evaluate the SDRS performance in Section V-D, by comparing the solutions obtained from the evolutionary approach.

\section{Performance Evaluation}

In this part, the weighted sum of two objectives is considered as the performance metric (referring the explanation of Figure 7 in Section V-A). As shown in Figure 7, the limit of the achievable weighted sum can be approximated by the evolutionary algorithm (i.e. the MOGA implementation as referred to in Section IV-B). From the obtained limit, the four schemes based on the greedy algorithm are evaluated given different weight values for the two objectives. Based on previous analysis and discussion, a small main budget (500 seconds in our case) is considered in these simulations and the extra budget is the control variable. Default settings are adopted for other factors $(\eta=0.9, \sigma=5, J=20)$ and the weight of the cost reduction is varied from 0 to 1 in 0.1 steps.

Figure 15 shows how the weighted sum varies with the weight value. Referring to the previous discussion of Figure 7, the convex curve obtained from the MOGA reflects a performance limit of the weighted sum and therefore this MOGA curve is considered as the optimum. DBRS-S is far from the optimum when the weight is zero, as it does not achieve a good delivery satisfaction. However, it improves with increasing weight and finally outperforms the other three greedy schemes when the weight is one. The remaining three greedy schemes are near the performance limit when the weight is small. With 
increasing weight value, DBRS-N is the first to move away from the optimum. DBRS-F is more close because the main budget is considered for the cost reduction. SDRS is closest to the limit as it can exploit more opportunities for cost reduction without notably influencing the delivery satisfaction.

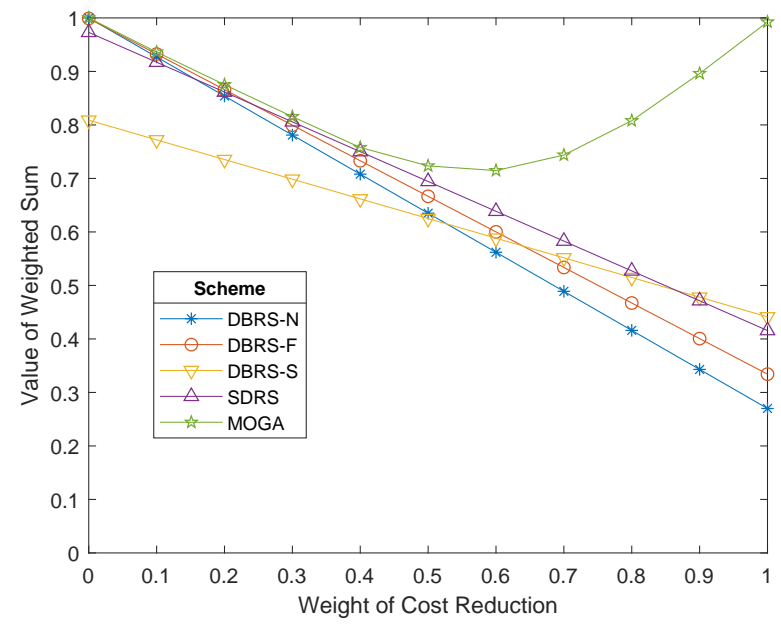

Fig. 15. Evaluation of weighted sum over different weight values (when main budget is 500s and extra budget is $1000 \mathrm{~s}$ )

Figure 16 further demonstrates the difference from the performance limit (i.e. the MOGA curve) for the four greedy schemes. This figure shows that when the weight value is small (less than 0.3 in this case), the greedy schemes generally have good performance (i.e. a small difference from the optimum) except DBRS-S which considers the second-level budget boundary and therefore introduces more uncertainty. With increasing weight to the cost reduction factor (especially when larger than 0.7 in this case), relay selection based on the greedy algorithm becomes no longer suitable. It is worth noting that SDRS outperforms both DBRS-F and DBRS-N when the weight value is larger than 0.3 , which confirms that our proposed SDRS scheme can find a better solution when the delivery efficiency becomes similarly (or more) important relative to the delivery satisfaction.

Figure 17 provides another view of how the extra budget affects the difference from the performance limit. It is shown that neither DBRS-N and DBRS-S yield good performance when there is an extra budget. With increasing extra budget, the difference of DBRS-F increases while the difference of SDRS decreases due to awareness of extra delay budget. As a conclusion, the proposed SDRS scheme can exploit the extra budget and therefore bring better performance.

\section{CONCLUSION}

In this paper, the relay selection problem is investigated for efficient group delivery, aimed at the IoT application scenarios that involve directional movement with randomness (such as animals in habitat monitoring). Following a review of the current limitations, an analytical framework is proposed and a combinatorial bi-objective optimisation problem is formulated. To solve the formulated problem, group relay selection is

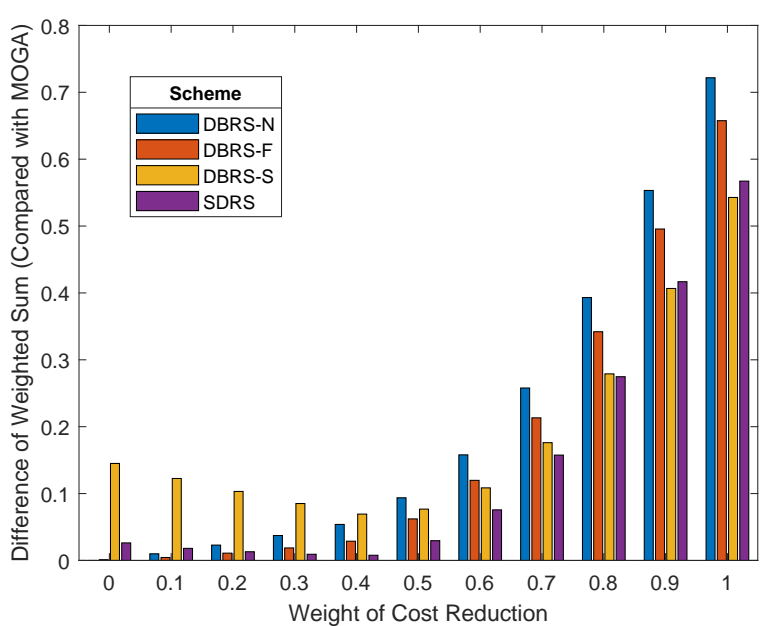

Fig. 16. Difference of weighted sum over different weight values (averaged for extra budget varying from $0 \mathrm{~s}$ to $3000 \mathrm{~s}$ in 500 s steps where main budget is $500 \mathrm{~s}$ )

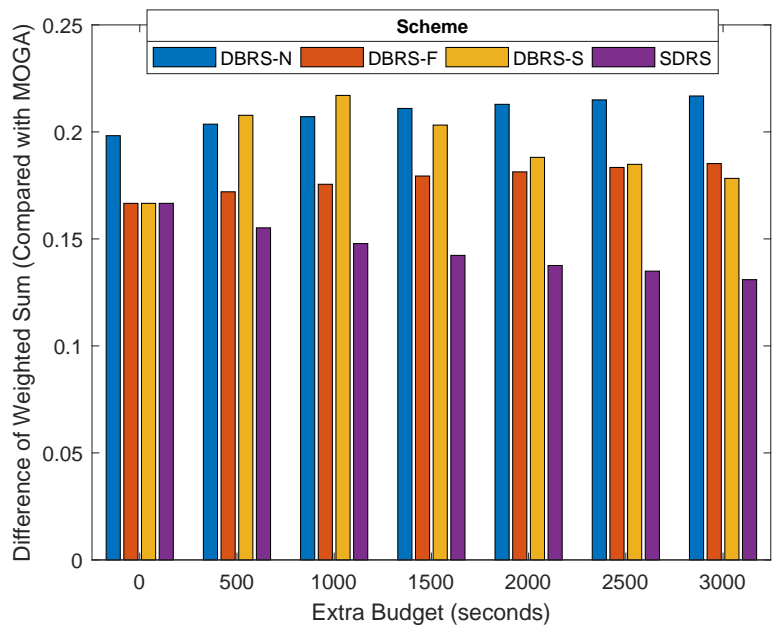

Fig. 17. Difference of weighted sum over different extra budget values (averaged for weight values varying from 0 to 1 in 0.1 steps where main budget is 500 s)

examined on the basis of an evolutionary approach and two kinds of greedy methods. In addition, a scenario with an extra delay budget is considered which reflects more flexibility of acceptable delay and brings about more opportunities for optimisation. Simulation results show that relay selection based on the greedy algorithm can achieve near-optimal performance when the delivery satisfaction is highly desirable. However, the performance of the greedy approach gradually becomes far from optimal with increasing weight given to delivery cost reduction. For cases where delivery efficiency becomes similarly (or more) important than delivery reliability, our proposed SDRS scheme is shown to be capable of finding more suitable solutions, which results in better delivery performance.

\section{REFERENCES}

[1] S. Poslad, Ubiquitous computing: smart devices, environments and interactions. John Wiley \& Sons, 2011. 
[2] Y. Lin, Y. Lin, C. Hsiao, and Y. Wang, "Iottalk-rc: Sensors as universal remote control for aftermarket home appliances," IEEE Internet of Things Journal, vol. 4, no. 4, pp. 1104-1112, Aug 2017.

[3] G. Xu, E. C. . Ngai, and J. Liu, "Ubiquitous transmission of multimedia sensor data in internet of things," IEEE Internet of Things Journal, vol. 5, no. 1, pp. 403-414, Feb 2018.

[4] N. H. Motlagh, T. Taleb, and O. Arouk, "Low-altitude unmanned aerial vehicles-based internet of things services: Comprehensive survey and future perspectives," IEEE Internet of Things Journal, vol. 3, no. 6, pp. 899-922, Dec 2016.

[5] M. Stecca, C. Moiso, M. Fornasa, P. Baglietto, and M. Maresca, "A platform for smart object virtualization and composition," IEEE Internet of Things Journal, vol. 2, no. 6, pp. 604-613, Dec 2015.

[6] X. Liu, T. Wei, and A. Liu, "Fast program codes dissemination for smart wireless software defined networks," Scientific Programming, p. 21, 2016.

[7] A. olakovi and M. Hadiali, "Internet of things (iot): A review of enabling technologies, challenges, and open research issues," Computer Networks, vol. 144, pp. $17-39,2018$.

[8] Y. Cao and Z. Sun, "Routing in delay/disruption tolerant networks: A taxonomy, survey and challenges," IEEE Communications Surveys Tutorials, vol. 15, no. 2, pp. 654-677, Second 2013.

[9] S. Batabyal and P. Bhaumik, "Mobility models, traces and impact of mobility on opportunistic routing algorithms: A survey," IEEE Communications Surveys Tutorials, vol. 17, no. 3, pp. 1679-1707, thirdquarter 2015.

[10] M. T. Nuruzzaman and H. W. Ferng, "A low energy consumption routing protocol for mobile sensor networks with a path-constrained mobile sink," in 2016 IEEE International Conference on Communications (ICC), May 2016, pp. 1-6.

[11] G. L. Chiou, S. R. Yang, and W. T. Yen, "On trajectory-based i2v group message delivery over vehicular ad-hoc networks," IEEE Transactions on Vehicular Technology, vol. 65, no. 9, pp. 7389-7402, Sept 2016.

[12] Y. Yao, Y. Sun, C. Phillips, and Y. Cao, "Movement-aware relay selection for delay-tolerant information dissemination in wildlife tracking and monitoring applications," IEEE Internet of Things Journal, vol. 5, no. 4, pp. 3079-3090, Aug 2018.

[13] C.-M. Yu, C.-S. Lu, and S.-Y. Kuo, "Habitual behavior-based opportunistic data forwarding in wildlife tracking," in 2007 4th International Symposium on Wireless Communication Systems. IEEE, 2007, pp. 807808.

[14] M. Grossglauser and D. N. C. Tse, "Mobility increases the capacity of ad hoc wireless networks," IEEE/ACM Transactions on Networking, vol. 10, no. 4, pp. 477-486, Aug 2002.

[15] A. Vahdat and D. Becker, "Epidemic routing for partially-connected ad hoc networks," 062000.

[16] T. Spyropoulos, K. Psounis, and C. S. Raghavendra, Efficient routing in intermittently connected mobile networks: the multiple-copy case. IEEE Press, 2008

[17] Y. Wang, X. Li, and J. Wu, "Multicasting in delay tolerant networks: Delegation forwarding," in 2010 IEEE Global Telecommunications Conference GLOBECOM 2010, Dec 2010, pp. 1-5.

[18] Y. Cao, K. Wei, G. Min, J. Weng, X. Yang, and Z. Sun, "A geographic multicopy routing scheme for dtns with heterogeneous mobility," IEEE Systems Journal, vol. PP, no. 99, pp. 1-12, 2017.

[19] Y. Li, D. Jin, P. Hui, and S. Chen, "Contact-aware data replication in roadside unit aided vehicular delay tolerant networks," IEEE Transactions on Mobile Computing, vol. 15, no. 2, pp. 306-321, Feb 2016

[20] R. Kim, H. Lim, and B. Krishnamachari, "Prefetching-based data dissemination in vehicular cloud systems," IEEE Transactions on Vehicular Technology, vol. 65, no. 1, pp. 292-306, Jan 2016.

[21] C. H. Papadimitriou and K. Steiglitz, Combinatorial Optimization: Algorithms and Complexity. Upper Saddle River, NJ, USA: PrenticeHall, Inc., 1982

[22] T. H. Cormen, C. E. Leiserson, R. L. Rivest, and C. Stein, Introduction to algorithms. MIT press, 2009.

[23] T. Wang, Y. Cao, Y. Zhou, and P. Li, "A survey on geographic routing protocols in delay/disruption tolerant networks," International Journal of Distributed Sensor Networks, vol. 12, no. 2, p. 3174670, 2016.

[24] A. Cerpa, J. Elson, D. Estrin, L. Girod, M. Hamilton, and J. Zhao, "Habitat monitoring: Application driver for wireless communications technology," ACM SIGCOMM Computer Communication Review, vol. 31, no. 2 supplement, pp. 20-41, 2001.

[25] B. N. Clark, C. J. Colbourn, and D. S. Johnson, "Unit disk graphs," Discrete Mathematics, vol. 86, no. 1, pp. 165 - 177, 1990.

[26] N. Gershenfeld, The nature of mathematical modeling. Cambridge University Press, 1999.
[27] E. A. Codling, M. J. Plank, and S. Benhamou, "Random walk models in biology," Journal of the Royal Society Interface, vol. 5, no. 25, pp. 813-834, 2008

[28] Y. Censor, "Pareto optimality in multiobjective problems," Applied Mathematics and Optimization, vol. 4, no. 1, pp. 41-59, 1977.

[29] K. Deb and D. Kalyanmoy, Multi-Objective Optimization Using Evolutionary Algorithms. New York, NY, USA: John Wiley \& Sons, Inc., 2001.

[30] K. Sohraby, D. Minoli, and T. Znati, Wireless sensor networks: technology, protocols, and applications. John Wiley \& Sons, 2007. 